\title{
Indian Spices and Biotherapeutics in Health and Chronic Disease
}

\author{
Ian James Martins ${ }^{1,2,3,4}$ \\ ${ }^{1}$ Centre of Excellence in Alzheimer's Disease Research and Care, Sarich Neuroscience Research Institute, Edith Cowan University, \\ Nedlands, Australia \\ ${ }^{2}$ School of Psychiatry and Clinical Neurosciences, The University of Western Australia, Nedlands, Australia \\ ${ }^{3}$ McCusker Alzheimer's Research Foundation, Hollywood Medical Centre, Nedlands, Australia \\ ${ }^{4}$ School of Medical and Health Sciences, Edith Cowan University, Nedlands, Australia
}

Email:i.martins@ecu.edu.au

How to cite this paper: Martins, I.J. (2018) Indian Spices and Biotherapeutics in Health and Chronic Disease. Health, 10, 374-380.

https://doi.org/10.4236/health.2018.104030

Received: March 9, 2018

Accepted: April 8, 2018

Published: April 11, 2018

Copyright $\odot 2018$ by author and Scientific Research Publishing Inc. This work is licensed under the Creative Commons Attribution International License (CC BY 4.0).

http://creativecommons.org/licenses/by/4.0/

\begin{abstract}
The acceleration in the rate of chronic disease that involves insulin resistance has become of concern in various countries. The rate of the most prevalent chronic diseases involves the metabolic syndrome and non alcoholic fatty liver disease (NAFLD) that is closely associated to diabetes and neurodegenerative diseases. Biotherapeutics and nutritional biotherapy have become important to reverse these global diseases. Biotherapeutics that involves Indian spice therapy requires assessment with relevance to insulin therapy, immunotherapy, antimicrobial therapy and drug therapeutics. Combined insulin therapy and Indian spice therapy regulates human insulin biological activity with relevance to the prevention of uncontrolled intracellular glucose levels and mitochondrial apoptosis. Biotherapeutics with nutritional biotherapy that involves the use of various nutrients such as magnesium and phosphatidylinositol (gm/day) is essential to insulin therapy. Factors such as stress, core body temperature and food quality influence biotherapeutics and Indian spice therapy with delayed spice clearance associated with mitochondrial dysfunction (cell apoptosis) and altered drug/caffeine therapy with relevance to the global diabetes pandemic.
\end{abstract}

\section{Keywords}

Spices, Biotherapeutics

\section{Editorial}

Biotherapeutics has become of importance to global chronic diseases to prevent accelerated aging associated with uncontrolled immune reactions that determine 
treatment and disease progression. In the global burden of disease connections between nutritional therapy and the immune system [1] [2] [3] have become of medical interest with primary immune dysregulation related to obesity, diabetes and neurodegenerative diseases. Nutritional diets are essential to maintain immunotherapy/antimicrobial therapy [2] [4] [5] [6] relevant to community factors and global antibiotic resistance [7] [8]. Drug biotherapeutics is essential to stabilize chronic disease with dietary interventions and fat consumption that determine biotherapeutics important to the treatment of endocrine and metabolic diseases. Nutritional interventions with Indian spices [9] [10] and insulin therapy [11] [12] (Figure 1) have become of critical importance to the global diabetic pandemic with human insulin and its biological activity (Figure 1) of major relevance to the global burden of disease progression.

Indian spices have been reported to exhibit a wide range of physiological and pharmacological properties that produce beneficial health promoting/protective effects for various chronic diseases [13]-[19]. Identification of spices such as five commonly used dietary spices include saffron, curcumin, pepper family, zingiber and cinnamon has been used for the treatment of hypercholesterolemic, cardiovascular disease, obesity, inflammation/metabolic disease, diabetes and Alzheimer's disease [13]-[19]. Indian spices as a biotherapy have become important in the developed and developing world with specific spices such as cinnamon and curcumin involved in the control of the immune system and the antimicrobial therapy [20] [21] [22] [23]. Cinnamon and curcumin as nutritional interventions have major effects on drug and hormone biotherapy with doses of these spices [9] important to determine stabilization and reversal of global chronic disease. Insulin therapy is one of the most important treatments in diabetes with

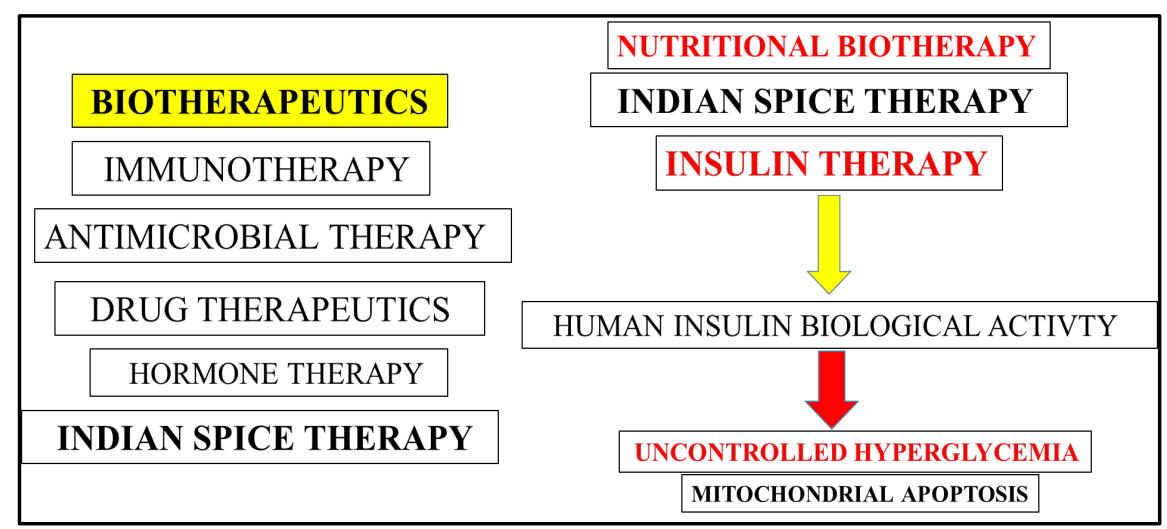

Figure 1. Biotherapeutics and nutritional biotherapy have become important to reverse global diseases such as non alcoholic fatty liver disease (NAFLD), diabetes and neurodegenerative diseases. Biotherapeutics that involve Indian spice therapy require assessment with relevance to immunotherapy, antimicrobial therapy and drug therapeutics. Combined insulin therapy and Indian spice therapy regulate human insulin biological activity with relevance to intracellular hyperglycemia and mitochondrial apoptosis. Databases searched for medical literature in this study include Pubmed database, Medline database, Research Gate Researcher Network, Mendeley Research Network and Academia.edu database. 
cinnamon and curcumin involved in the improvement of plasma hyperglycemia and involved with the regulation of insulin dose/type and frequency of use in diabetes therapeutics [24] [25] [26] [27]. Cinnamon has been shown to regulate insulin levels [24] [25] with therapeutic effects on hyperglycemia induced mitochondrial apoptosis [26]. Curcumin effects on the insulin receptor and beta cell function [26] [27] modulates human insulin therapy with critical consideration of Indian spice therapy required with relevance to human insulin administration and diabetes treatment [28]. Diabetes and mitochondrial dysfunction are closely connected [1] [29] with Indian spice and Insulin therapy to be carefully assessed with relevance combined therapy and increased cellular glucose levels related to hyperglycemic mitochondrial apoptosis [30].

Biotherapeutics for diseases (Figure 2) are now ineffective with malfunction of nutrient sensitive genes involved in mitochondrial survival [1] [3] [4] [30]. Indian spices (curcumin) as a biotherapy in health and disease should be carefully controlled with higher doses not associated with activation of anti-aging genes [31] involved in mitochondrial biogenesis [3]. Biotherapeutics that involve nutritional biotherapy with phosphatidylinositol (gm/day) [4] and magnesium contents [32] improves insulin therapy but combined therapy with Indian spices need to be assessed in clinical trials. Biotherapeutics that include other nutrients [33] are essential for biotherapy to maintain genomic stability in diabetes (Figure 2). Foods that contain essential nutrients include protein, eggs, cottage cheese, dairy, red meat, chicken, legumes, duck, nuts, and seeds. These essential nutrients include methionine, methylsulfonylmethane, sulphur, choline, and trimethylglycine as building blocks that allow regulation of genes by appropriate telomeres. Vitamins such as vitamin B12, folic acid, and vitamin B6 play multiple

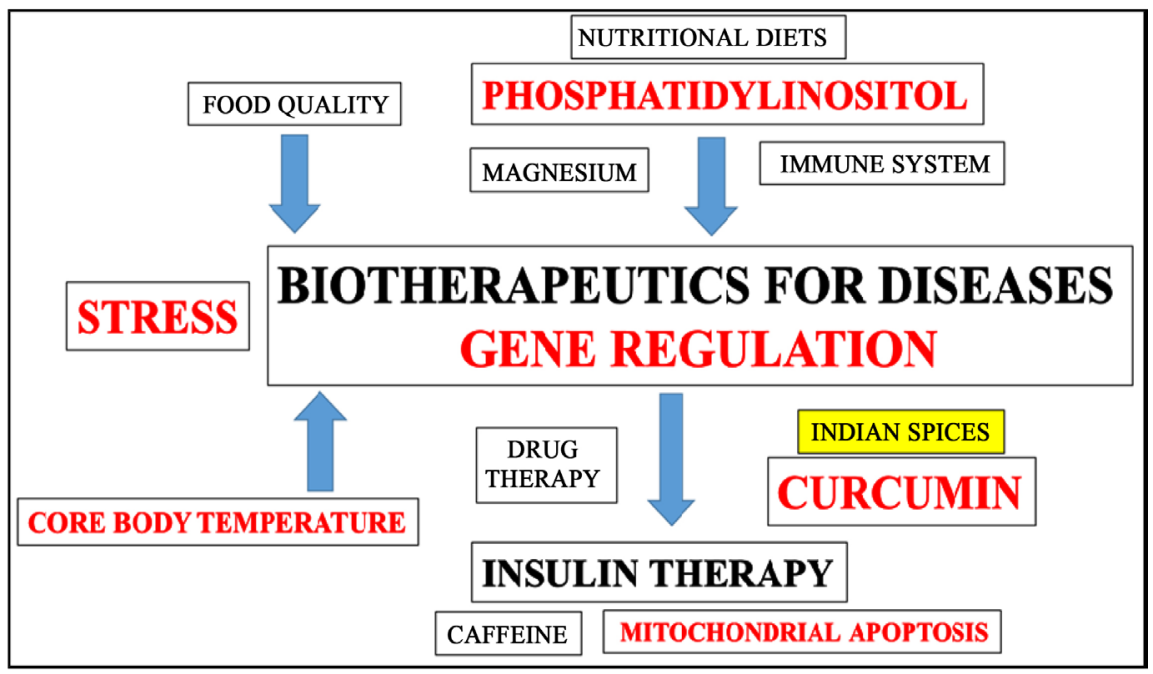

Figure 2. Biotherapeutics with nutritional biotherapy involve the use of various nutrients such as magnesium and phosphatidylinositol (gm/day) are essential to maintain genes involved in insulin therapy. Factors such as stress, core body temperature and food quality influence biotherapeutics and Indian spice therapy with delayed spice clearance associated with mitochondrial dysfunction (cell apoptosis) and altered drug/caffeine therapy. 
roles in genomic stability. Antioxidants and vitamins C, D and E are essential and maintain genomic stability. A lack of antioxidants leads to increased free radical damage and more risk for damage to telomeres essential to cell survival. Minerals such as magnesium and zinc are required for the prevention of DNA strand breakage and the prevention of accelerated cell aging. Nutrients such as quercetin, green tea catechins, grape seed extract, resveratrol and omega 3 fatty acids (eicosapen-taenoic acid/docosahexaenoic acid) are important as basic nutrients to preserve biological aging and reverse diabetes. Poor food quality [4] [5] [34] interferes with drug biotherapeutics associated with Indian spice inactivation (Figure 2). Biotherapeutics that involves caffeine has been extensively studied in obesity and diabetes [35] [36] with curcumin doses [10] and caffeine intake important to hyperglycemia induced cell apoptosis. Indian spices that induce cell apoptosis [37] [38] [39] prevent cancer include curcumin (turmeric) and piperine (black pepper) with interference with caffeine metabolism [40] and active spice component pharmacokinetic data is still not available.

Core body temperature connections to the immune system and mitochondrial cell function [41] indicate that with heat/cold stress induce toxic immune reactions [42] that are relevant to mitochondrial apoptosis in non alcoholic fatty liver disease, obesity, diabetes and neurodegenerative diseases. Factors such as core body temperature and stress [41] [43] may override Indian spice therapy and various biotherapeutics that are of prime importance in the stabilization of the global chronic disease epidemic [31] [44]. Indian spice therapy requires further assessment with relevance to hormone therapy, reversal of NAFLD with poor Indian spice metabolism possibly relevant to adverse drug reactions [9] (Indian spice-drug interactions) with the aging process but higher doses are therapeutic for cancer treatment with relevance to induction of cell apoptosis [29] [30] [31]. Furthermore diets that contain Indian spices may alter the apelinergic system [43] involved in stress reactions, co-ordination of the neuroendocrine system and the development of chronic disease.

\section{Conclusion}

Biotherapeutics for chronic diseases has accelerated to prevent the progression of the current global chronic disease epidemic. Indian spice therapy has become an important biotherapeutic involved in the reversal of global diabetes and neurodegeneration. Mitophagy in chronic disease requires attention with Indian spice therapy and insulin therapy as a combined therapy to regulate cell glucose levels to prevent hyperglycemic induced mitochondrial apoptosis. Specific nutrients need to be consumed with Indian spices to allow stabilization of uncontrolled toxic reactions that lead to cell death. Core body temperature, stress and inappropriate food quality will inactivate Indian spice therapy with excessive Indian spice intake over many years that may be connected to ineffective human insulin biological activity/drug biotherapeutics with long term Indian spice use more relevant to cell apoptosis and the treatment of cancer. Monitoring of long 
term Indian spice therapy may be required in future clinical trials in man with relevance to safety compared to diabetic individuals with insulin therapy and without Indian spice therapy.

\section{Acknowledgements}

This work was supported by grants from Edith Cowan University, the McCusker Alzheimer's Research Foundation and the National Health and Medical Research Council.

\section{References}

[1] Martins, I.J. (2017) Autoimmune Disease and Mitochondrial Dysfunction in Chronic Diseases. Research on Chronic Disease, 1, 10-12.

[2] Martins, I.J. (2017) Appetite Control and Nutrigenomic Diets Are Connected to Immune Regulation and Diabetes Prevention. EC Nutrition, 12, 120-123.

[3] Martins, I.J. (2015) Unhealthy Nutrigenomic Diets Accelerate NAFLD and Adiposity in Global communities. Journal of Molecular and Genetic medicine, 9, 1-8.

[4] Martins, I.J. (2015) Overnutrition Determines LPS Regulation of Mycotoxin Induced Neurotoxicity in Neurodegenerative Diseases. International Journal of Molecular Science, 16, 29554-29573. https://doi.org/10.3390/ijms161226190

[5] Martins, I.J. (2016) Food and Nutrition Cause Liver and brain Diseases. https://atlasofscience.org/food-and-nutrition-cause-liver-and-brain-diseases-with-d iabe

[6] Martins, I.J. (2017) Antimicrobial Activity Inactivation and Toxic Immune Reactions Induce Epilepsy in Human. Journal of Medical Discovery, 2, 1-7.

[7] Larson, E. (2007) Community Factors in the Development of Antibiotic Resistance. Annual Reviews in Public Health, 28, 435-447. https://doi.org/10.1146/annurev.publhealth.28.021406.144020

[8] Martins, I.J. (2017) Antibiotic Resistance Involves Antimicrobial Inactivation in Global Communities. Scholarena Journal of Pharmacy and Pharmacology, 2, 1-3.

[9] Martins, I.J. (2018) Indian Spices and Unhealthy Diets Interfere with Drug Therapy in Diabetes and Neurodegenerative Diseases. Novel Approaches in Drug Designing and Development, 3, 1-4.

[10] Martins, I.J. (2018) Indian Spices and Caffeine Treatment for Obesity and Cardiovascular Disease. Annals of Clinical Endocrinology and Metabolism, 2, 10-14.

[11] Martins, I.J. (2017) Insulin Therapy Inactivation Is Connected to NAFLD and Diabetes Severity Index. Journal of Diabetes and Clinical Studies, 1, 1-3.

[12] Martins, I.J. (2018) Indian Spices and Insulin Therapy in Diabetes and Neurodegenerative Diseases. Journal of Diabetes and Clinical Studies, 1, 1-3.

[13] Krishnaswamy, K (2008) Traditional Indian Spices and Their Health Significance. Asia Pacific Journal of Clinical Nutrition, 217, 265-268.

[14] Iyer, A., Panchal, S., Poudyal, H. and Brown, L (2009) Potential Health Benefits of Indian Spices in the Symptoms of the Metabolic Syndrome: A Review. Indian Journal of Biochemistry \& Biophysics, 46, 467-481.

[15] Vasanthi, H.R. and Parameswari, R.P. (2010) Indian Spices for Healthy Heart-An Overview. Current Cardiology Reviews, 6, 274-279.

https://doi.org/10.2174/157340310793566172 
[16] Aggarwal, B.B. (2010) Targeting Inflammation-Induced Obesity and Metabolic Diseases by Curcumin and Other Nutraceuticals. Annual Review of Nutrition, 30, 173-199. https://doi.org/10.1146/annurev.nutr.012809.104755

[17] Mishra, S. and Palanivelu, K. (2008) The Effect of Curcumin (Turmeric) on Alzheimer's Disease: An Overview. Annals of Indian Academy of Neurology, 11, 13-19. https://doi.org/10.4103/0972-2327.40220

[18] Reddy, P.H., Manczak, M., Yin, X., Grady, M.C., Mitchell, A., Tonk, S., et al. (2018) Protective Effects of Indian Spice Curcumin against Amyloid Beta in Alzheimer's Disease. Journal of Alzheimer's Disease, 61, 843-866. https://doi.org/10.3233/JAD-170512

[19] Sun, D., Lv, J., Chen, W., Li, S., Guo, Y., Bian, Z., et al. (2014) Spicy Food Consumption Is Associated with Adiposity Measures among Half a Million Chinese People: The China Kadoorie Biobank Study. BMC Public Health, 14, 1293. https://doi.org/10.1186/1471-2458-14-1293

[20] Nabavi, S.F., Lorenzo, A.D, Izadi, M., Sobarzo-Sánchez, E., Daglia, M. and Nabavi, S.M. (2015) Antibacterial Effects of Cinnamon: From Farm to Food, Cosmetic and Pharmaceutical Industries. Nutrients, 7, 7729-7748. https://doi.org/10.3390/nu7095359

[21] Roth-Walter, F., Moskovskich, A., Gomez-Casado, C., Diaz-Perales, A., Oida, K., Singer, J., Kinaciyan, T., Fuchs, H.C. and Jensen-Jarolim, E. (2014) Immune Suppressive Effect of Cinnamaldehyde Due to Inhibition of Proliferation and Induction of Apoptosis in Immune Cells: Implications in Cancer. PLoS ONE, 9, e108402. https://doi.org/10.1371/journal.pone.0108402

[22] Gunes, H., Gulen, D., Mutlu, R., Gumus, A., Tas, T. and Topkaya, A.E. (2016) Antibacterial Effects of Curcumin: An in Vitro Minimum Inhibitory Concentration Study. Toxicology and Industrial Health, 32, 246-250.

https://doi.org/10.1177/0748233713498458

[23] Bose, S., Panda, A.K., Mukherjee, S. and Sa, G. (2015) Curcumin and Tumor Immune-Editing: Resurrecting the Immune System. Cell Division, 10, 6. https://doi.org/10.1186/s13008-015-0012-Z

[24] Qin, B., Panickar, K.S. and Anderson, R.A. (2010) Cinnamon: Potential Role in the Prevention of Insulin Resistance, Metabolic Syndrome, and Type 2 Diabetes. Journal of Diabetes and Science Technology, 4, 685-693. https://doi.org/10.1177/193229681000400324

[25] Anderson, R.A., Zhan, Z., Luo, R., Guo, X., Guo, Q., Zhou, J., Kong, J., Davis, P.A. and Stoecker, B.J. (2015) Cinnamon Extract Lowers Glucose, Insulin and Cholesterol in People with Elevated Serum Glucose. Journal of Traditional and Complementary Medicine, 6, 332-336. https://doi.org/10.1016/j.jtcme.2015.03.005

[26] Jiménez-Osorio, A.S., Monroy, A. and Alavez, S. (2016) Curcumin and Insulin Resistance-Molecular Targets and Clinical Evidences. Biofactors, 42, 561-580. https://doi.org/10.1002/biof.1302

[27] Chuengsamarn, S., Rattanamongkolgul, S., Luechapudiporn, R., Phisalaphong, C. and Jirawatnotai, S. (2012) Curcumin Extract for Prevention of Type 2 Diabetes. Diabetes Care, 35, 2121-2127. https://doi.org/10.2337/dc12-0116

[28] Vantyghem, M.C., Defrance, F., Quintin, D., Leroy, C., Raverdi, V. and Prévost, G. (2014) Treating Diabetes with Islet Transplantation: Lessons from the Past Decade in Lille. Diabetes and Metabolism, 40, 108-119. https://doi.org/10.1016/j.diabet.2013.10.003

[29] Martins, I.J. (2018) Genomic Medicine and Acute Cardiovascular Disease Progres- 
sion in Diabetes. Research on Chronic Diseases, 2, 1-3.

[30] Cai, L., Li, W., Wang, G., Guo, L., Jiang, Y. and Kang, Y.J. (2002) Hyperglycemia-Induced Apoptosis in Mouse Myocardium: Mitochondrial Cytochrome C-Mediated Caspase-3 Activation Pathway. Diabetes, 51, 1938-1948. https://doi.org/10.2337/diabetes.51.6.1938

[31] Martins, I.J. (2016) Anti-Aging Genes Improve Appetite Regulation and Reverse Cell Senescence and Apoptosis in Global Populations. Advances in Aging Research, 5, 9-26. https://doi.org/10.4236/aar.2016.51002

[32] Martins, I.J. (2016) Magnesium Therapy Prevents Senescence with the Reversal of Diabetes and Alzheimer's Disease. Health, 8, 694-710. https://doi.org/10.4236/health.2016.87073

[33] Martins, I.J., Lim, W.L., Wilson, A., Laws, S. and Martins, R.N. (2013) The Acceleration of Aging and Alzheimer's Disease through the Biological Mechanisms behind Obesity and Type II Diabetes. Health, 5, 913-920. https://doi.org/10.4236/health.2013.55121

[34] Martins, I.J. (2016) Food Quality Induces a Miscible Disease with Relevance to Alzheimer's Disease and Neurological Diseases. Journal of Food Research, 5, 45-52. https://doi.org/10.5539/jfr.v5n6p45

[35] Lane, J.D., Barkauskas, C.E., Surwit, R.S. and Feinglos, M.N. (2004) Caffeine Impairs Glucose Metabolism in Type 2 Diabetes. Diabetes Care, 27, 2047-2048. https://doi.org/10.2337/diacare.27.8.2047

[36] Shi, X., Xue, W., Liang, S., Zhao, J. and Zhang, X. (2016) Acute Caffeine Ingestion Reduces Insulin Sensitivity in Healthy Subjects: A Systematic Review and Meta-Analysis. Nutrition Journal, 15, 103. https://doi.org/10.1186/s12937-016-0220-7

[37] Zheng, J., Zhou, Y., Li, Y., Xu, D.-P., Li, S. and Li, H.-B. (2016) Spices for Prevention and Treatment of Cancers. Nutrients, 8, E495. https://doi.org/10.3390/nu8080495

[38] Kaefer, C.M. and Milner, J.A. (2011) Herbs and Spices in Cancer Prevention and Treatment. In: Benzie, I.F.F. and Wachtel-Galor, S., Eds., Herbal Medicine. Biomolecular and Clinical Aspects, 2nd Edition, CRC Press/Taylor \& Francis, Boca Raton, Chapter 17.

[39] Alam, I., Imam, H. and Riaz, Z. (2015) Cancer Preventing Spices. Journal of Cancer Metastasis and Treatment, 1, 41-42.

[40] Martins, I.J. (2017) Caffeine with Links to NAFLD and Accelerated Brain Aging. In: Valenzuela, R., Ed., Non-Alcoholic Fatty Liver Disease-Molecular Bases, Prevention and Treatment, InTechOpen, London, Chapter 8.

[41] Martins, I.J. (2018) Regulation of Body Temperature and NAFLD in Global Population.

[42] Martins, I.J. (2017) Regulation of Core Body Temperature and the Immune System Determines Species Longevity. Current Updates in Gerontology, 1, 1-6.

[43] Martins, I.J. (2015) Nutritional Diets Accelerate Amyloid Beta Metabolism and Prevent the Induction of Chronic Diseases and Alzheimer's Disease. Photon ebooks, $1-48$.

[44] Martins, I.J. (2014) Induction of NAFLD with Increased Risk of Obesity and Chronic Diseases in Developed Countries. Open Journal of Endocrine and Metabolic Diseases, 4, 90-110. https://doi.org/10.4236/ojemd.2014.44011 